\title{
Diversidade de anfíbios do Estado de Mato Grosso do Sul, Brasil
}

\author{
Franco L. Souza', Cynthia P.A. Prado², José L.M.M. Sugai³, Vanda L. Ferreira', \\ Camila Aoki ${ }^{4}$, Paulo Landgref-Filho ${ }^{4}$, Christine Strüssmann ${ }^{5}$, \\ Robson W. Ávila ${ }^{6}$, Domingos J. Rodrigues ${ }^{7}$, Nelson R. Albuquerque?, \\ Juliana Terra 3 , Masao Uetanabaro ${ }^{8}$, Arlindo F. Béda ${ }^{4}$, Liliana Piatti', \\ Ricardo A. Kawashita-Ribeiro9, Milena Delatorre3 ${ }^{3}$, Gabriel P. Faggioni ${ }^{3}$, \\ Stephani D.B. Demczuk ${ }^{3}$ \& Samuel Duleba ${ }^{10}$
}

\footnotetext{
1. Universidade Federal de Mato Grosso do Sul, Centro de Ciências Biológicas e da Saúde, Caixa Postal 549, 79070-900 Campo Grande, MS, Brasil. (franco.souza@ufms.br)

2. Universidade Estadual Paulista Júlio de Mesquita Filho, Faculdade de Ciências Agrárias e Veterinárias de Jaboticabal, Via de Acesso Prof. Paulo Donato Castellane Km 05 - Dep. de Morfologia e Fisiologia Animal. 14884-900 Jaboticabal, SP, Brasil.

3. Universidade Federal de Mato Grosso do Sul, Centro de Ciências Biológicas e da Saúde, Programa de Pós-Graduação em Ecologia e Conservação, Campo Grande, MS, Brasil.

4. Universidade Federal de Mato Grosso do Sul, Campus Universitário de Aquidauana - CPAQ, Departamento de Biociências, Aquidauana, MS, Brasil.

5. Universidade Federal de Mato Grosso, Faculdade de Agronomia e Medicina Veterinária, Dep. de Ciências Básicas e Produção Animal, Av. Fernando Correia,

2367, 78060-900 Cuiabá, MT, Brasil.

6. Universidade Regional do Cariri, Departamento de Ciências Físicas e Biológicas, Rua Cel. Antonio Luiz, 63105-100 Crato, CE, Brasil.

7. Universidade Federal de Mato Grosso, Campus Universitário de Sinop/ICNHS, Av. Alexandre Ferrano, 1200, 78557-267 Sinop, MT, Brasil.

8. Universidade Federal de Mato Grosso do Sul, Campus do Pantanal, Av. Rio Branco, 1270, Caixa Postal 252, 79304-902 Corumbá, MS, Brasil.

9. Universidade Federal de Mato Grosso, Instituto de Biociências, Dep. de Ecologia, Pós-Graduação em Ecologia e Conservação da Biodiversidade, Cuiabá MT, Brasil.

10. Universidade Federal de Mato Grosso do Sul, Centro de Ciências Biológicas e da Saúde, Programa de Pós-Graduação em Biologia Animal, Campo Grande, MS, Brasil.
}

Recebido 1 dezembro 2016

Aceito 6 fevereiro 2017

DOI: $10.1590 / 1678-4766 e 2017152$

ABSTRACT. Amphibian diversity of Mato Grosso do Sul State, Brazil. Amphibian species from Mato Grosso do Sul state were compiled and checked for the first time. A total of 97 species were recorded, representing more than $10 \%$ of the known Brazilian amphibians. Twenty five anuran genera in nine families and only one caecilian were recorded. Cerrado was the richest area followed by semideciduous forests, Chaco, and veredas (palm swamps). It was recorded 56 species in floodplain areas and 92 in the surrounding cerrados. The Serra da Bodoquena National Park and some Pantanal areas were better sampled than other regions in Mato Grosso do Sul, resulting in sampling gaps across the state. Important areas for future inventories were identified: mountainous regions in Serra de Maracaju and those bordering the Apa river, Brazilian chacoan areas, the Taquari river alluvial fan, the broad Taquari-Itiquira flat area, the Central Plateau scarps, wet areas from Parana river bordering São Paulo state, and inland areas from Pantanal.

KEYWORDS. Amphibia, inventories, biodiversity, Biota-MS Program.

RESUMO. A fauna de anfíbios do estado de Mato Grosso do Sul foi compilada e elaborada pela primeira vez, totalizando 97 espécies, o que representa pouco mais de $10 \%$ das espécies que ocorrem no Brasil. Nove famílias e 25 gêneros de Anura e apenas uma espécie de Gymnophiona foram registradas. As áreas de Cerrado apresentaram a maior riqueza, seguidas pelas florestas estacionais semideciduais, Chaco e veredas. Em relação às macrorregiões consideradas, foram registradas 56 espécies para a planície de inundação (majoritariamente representada pelo Pantanal) e 92 para o planalto. As regiões mais amostradas em Mato Grosso do Sul são o Parque Nacional da Serra da Bodoquena e algumas áreas do Pantanal, o que resulta em lacunas de amostragens por todo o estado, principalmente nas regiões norte, leste/sudeste e parte do oeste/sudoeste. Foram identificadas áreas consideradas importantes para amostragens em decorrência da complexidade de fitofisionomias e relevos encontrados no estado: Maciço do rio Apa, Serra de Maracaju, grande parte do Chaco brasileiro, leque do rio Taquari, planalto do Taquari-Itiquira, escarpas do planalto Central, áreas úmidas/florestas estacionais da planície do rio Paraná e áreas interioranas do Pantanal, onde o acesso é difícil.

PALAVRAS-CHAVE. Amphibia, inventários, biodiversidade, Programa Biota-MS.

Mato Grosso do Sul é um estado jovem, tendo sido desmembrado do estado de Mato Grosso há 35 anos. Essa informação é importante quando se pretende considerar a atual condição do conhecimento para diversos segmentos, incluindo a biodiversidade. Em associação à idade, a grande extensão territorial $(\sim 360$ mil km²), aliada à paisagem complexa em termos de relevo, hidrologia e vegetação (FILHO et al., 2004; Godoy et al., 2009; GonÇALVES et al., 2011; Pотт et al., 2011), fazem do estado uma área ainda pouco explorada quanto à composição da biota. Não por acaso, são frequentes mapas de distribuição de espécies de anuros em que o Mato Grosso do Sul representa uma lacuna, mesmo quando há registros para estados vizinhos (e.g., AlmeIDA et al., 2008; Moura et al., 2010; ANDRADE \& VAZ-SiLva, 
2012). Apesar de vários trabalhos de inventários e descrições de espécies publicadas recentemente terem começado a preencher essas lacunas (AOKI et al., 2010, 2011; ÁvilA et al., 2010; AMARAL et al., 2012; LANDGREF-FilHo et al., 2012; Souza-Filho \& Lima, 2012; Sugai et al., 2012), o número de amostragens ainda é incipiente e diversas áreas do estado ainda necessitam ser inventariadas (ver revisão em STRÜSSMANN et al., 2007, 2011).

São reconhecidas para o Brasil 1026 espécies de anfíbios (SEgalla et al., 2014), sendo 988 Anura, uma espécie de Caudata e 33 de Gymnophiona. Apesar dessa riqueza, informações básicas como padrões de distribuição geográfica, de endemismo e dinâmica populacional ainda são escassas para muitas espécies, o mesmo sendo válido para estudos dessa natureza em diferentes hábitats ou fitofisionomias. Os registros de espécies de anfíbios para o estado de Mato Grosso do Sul são extremamente pontuais (ver Gordo \& Campos, 2003, 2005; Ávila \& Ferreira, 2004; StRÜSSMANN et al., 2007, para histórico de registros para o estado) e, em alguns casos, abrangem as áreas da planície de inundação e seus arredores, onde está inserida a grande região do Pantanal (PCBAP, 1997; STRÜSSMANN et al., 2000; GoRDO \& CAMPOS, 2003; WANG et al., 2005; UetanABARO et al., 2007, 2008; Souza et al., 2010).

Nos últimos anos, vários estudos têm propiciado um incremento de informações sobre os padrões de distribuição de anfíbios para diversas regiões do Brasil, avaliando também os condicionantes ecológicos destas distribuições (Siqueira et al., 2009; VASCONCElos et al., 2010; Silva et al., 2011; Toledo \& BATista, 2012; VAldujo et al., 2012a). Em particular, a produção de listas de espécies de diversas localidades é crucial tanto para indicar lacunas de conhecimento em áreas a serem inventariadas, como para subsidiar planos de manejo e estudos que possam indicar áreas prioritárias à conservação (e.g., DiNIz-FILHO et al., 2007; LOYOLA et al., 2008; BECKER et al., 2010; TOLEDO \& BATISTA, 2012; Verdade et al., 2012). O presente trabalho tem como objetivo apresentar uma lista das espécies de anfíbios do estado de Mato Grosso do Sul, suas regiões de ocorrência e as principais formações vegetais associadas aos registros.

\section{MATERIAL E MÉTODOS}

Os dados aqui apresentados reúnem informações oriundas de coleções científicas do estado (Coleção Zoológica de Referência da Universidade Federal de Mato Grosso do Sul, Campo Grande - ZUFMS e Coleção de Vertebrados do Pantanal, Embrapa Pantanal, Corumbá - HCPAP) e de outras instituições (Brasília, DF, Coleção Herpetológica da Universidade de Brasília, CHUNB; Goiânia, GO, Coleção Zoológica da Universidade Federal de Goiás, ZUFG; Rio Claro, SP, Célio F.B. Haddad, Instituto de Biociências, Universidade Estadual Paulista, CFBH; Curitiba, PR, Museu de História Natural Capão da Imbuia, MHNCI; Campinas, SP, Universidade Estadual de Campinas, ZUEC; Porto Alegre, RS, Museu de Ciências e Tecnologia da PUCRS, MCT -
Anfíbios; Departamento de Zoologia e Botânica de São José do Rio Preto, SP, Coleção de Anfíbios DZSJRP; Cuiabá, MT, Coleção Zoológica de Vertebrados da Universidade Federal de Mato Grosso, UFMT). Também foram considerados registros da literatura e informações pessoais de pesquisadores. Para cada registro foram identificadas as coordenadas geográficas (DATUM: WGS84) e, quando não disponíveis, estas foram aproximadas através de imagens do Google Earth ${ }^{\mathrm{TM}}$, as quais foram utilizadas para gerar mapas de registro de ocorrência das espécies através do programa Quantum GIS versão 1.8.0 (QuANTUM GIS DEVELOPMENT TEAM, 2012). Os mapas também representam as principais características do relevo, com base em dados obtidos através de depositórios específicos (WeBER et al., 2004), a vegetação e Unidades de Conservação de proteção integral criadas no estado até o ano de 2011 (http://www.ibge.gov.br/home/geociencias/ default_prod.shtm; http://www.ibama.gov.br/zoneamentoambiental).

De posse dos registros, utilizou-se o aplicativo DIVAGIS (Hijmans et al., 2002) que, através do método "circular neighborhood", gerou um mapa em formato rasterizado, indicando as regiões do estado com maior número de registros de coleta de anfíbios e as regiões menos amostradas, sendo as últimas consideradas como lacunas de conhecimento (RoSSA-FERES et al., 2011).

Localizado na porção central da América do Sul, Mato Grosso do Sul apresenta distintas formações com componentes florísticos particulares. Grande parte do estado está inserida no domínio fitogeográfico do Cerrado, constituído em sua maioria por cerrado sensu lato, o que inclui campo limpo, campo sujo e cerradão. Além dessas diversas fitofisionomias do cerrado, outros tipos vegetacionais como veredas de buritis e florestas estacionais semidecíduas (sensu BATALHA, 2011) também estão presentes. Já o domínio fitogeográfico do Chaco é representado por uma pequena extensão de área no sudoeste do estado, incluindo suas diferentes fitofisionomias de chaco arbóreo, gramíneo e arbustivo. Dessa maneira, para uma primeira tentativa de associação de anfíbios com as características florísticas regionais, os registros foram vinculados a esses principais domínios e aos seus tipos vegetacionais. Longe de ser uma classificação da flora do estado, as formações propostas aqui (Cerrado, veredas, florestas estacionais semidecíduas e Chaco) devem ser vistas como uma forma de regionalização da fauna de anfíbios do Mato Grosso do Sul. Ao mesmo tempo, os registros também foram associados com duas grandes formas de relevo que ocorrem no estado e delimitam duas macrorregiões: a planície inundável, composta majoritariamente pelas áreas do Pantanal, com suas diversas subdivisões (ADÁmoli, 1982) e o planalto. Para esse último, as regiões elevadas, localizadas sobre escarpas e "morrarias" (termo regional para designar a ocorrência de série de morros em determinado local), mesmo que inseridas na planície inundável, foram consideradas como planalto.

Em termos de conservação, as espécies de anfíbios registradas para o estado foram assinaladas segundo os critérios de ameaça propostos pela União Internacional para a 
Conservação da Natureza (IUCN, 2001), a saber: criticamente em perigo (CR), em perigo (EN) e vulnerável (VU). Embora as categorias dados insuficientes (DD) e de menor risco (LC) não estejam vinculadas a graus de ameaça, também foram incluídas por serem consideradas um reflexo do atual conhecimento das espécies, como já sugerido por VERDADE et al. (2012). A nomenclatura utilizada segue AMPHIBIAWEB (2012), a qual está atualizada segundo revisões recentes (FAivovich et al., 2005; NAscimento et al., 2006; Hedges et al., 2008; NARVAEs \& Rodrigues, 2009; GARDA et al., 2010; Blackburn \& WaKe, 2011; Kolenc et al., 2011; Pyron \& Wiens, 2011; Wilkison et al., 2011; Padial et al., 2012). Seguimos Lavilla et al. (2010) na nomenclatura de Trachycephalus typhonius.

\section{RESULTADOS E DISCUSSÃO}

De acordo com os dados disponíveis até o momento, foram registradas 97 espécies de anfíbios para o Mato Grosso do Sul, das quais nove aguardam confirmação, o que representa pouco mais de $10 \%$ das espécies de anfíbios do Brasil. Apenas uma espécie de Gymnophiona foi registrada (Siphonops paulensis), enquanto que as demais espécies pertencem à Ordem Anura, distribuídas entre nove famílias e 25 gêneros (Tab. I). As áreas com vegetação de cerrado sensu lato apresentaram a maior riqueza de espécies de anuros (70), seguidas pelas florestas estacionais semidecíduas (57), veredas (47) e Chaco (37). Naquelas áreas com forte influência antrópica, como pastagens, campos agrícolas e mesmo regiões urbanas, foram registradas 54 espécies (Tab. I).

A maior parte do território do Mato Grosso do Sul é constituída ou tem forte influência do Cerrado, o que explicaria a alta representatividade de espécies de anuros típicos desse domínio registrada no estado. O Cerrado abriga uma fauna de anuros estruturada espacialmente em resposta a fatores históricos e gradientes ambientais existentes em toda sua extensão, apresentando assim elevados valores de diversidade beta (DiNIZ-FiLHo et al., 2008; VALDUJo et al., 2012a). Atualmente são conhecidas 209 espécies de anuros para o Cerrado e áreas de contato (VALDUJo et al., 2012b), das quais $75(36 \%)$ foram registradas para Mato Grosso do Sul. O Cerrado é a maior, mais diversa e, talvez, a mais ameaçada savana tropical do mundo (MYers et al., 2000; SILVA \& BATES, 2002), sendo também o segundo maior domínio fitogeográfico brasileiro, com área original de 2,5 milhões de $\mathrm{km}^{2}$ (SiLVA et $a l$. , 2006). No entanto, o Cerrado tem sofrido intensa ação antrópica nas últimas décadas em decorrência da urbanização e desenvolvimento de atividades agropastoris. Estima-se que mais da metade da área originalmente ocupada pelo Cerrado tenha sido transformada nas últimas décadas em decorrência de diversas atividades antrópicas e, apesar de sua importância para a biodiversidade, menos de $2 \%$ dessa área encontra-se protegida (KLINK \& MACHADO, 2005).

Em relação às florestas estacionais, são formações importantes geralmente associadas a regiões com características geológicas particulares como bordas do planalto e solos calcários (Prado \& GibBS, 1993), como é o caso da Serra da Bodoquena e de regiões elevadas (morrarias) de Corumbá. Estas florestas encontram-se fortemente ameaçadas por atividades antrópicas, tais como mineração, exploração madeireira e abertura de pastagens (Miles et al., 2006; SiLva et al., 2006). Dada a peculiaridade desse ambiente e sua distribuição de forma isolada no estado, as florestas estacionais podem vir a representar áreas de endemismo em potencial, pois muitas localidades ainda necessitam de avaliação quanto à sua riqueza de fauna $\mathrm{e}$ flora (UETANABARO et al., 2007).

As veredas apresentaram a terceira maior riqueza de espécies de anfíbios no estado. Apesar de sua inestimável importância na manutenção da biodiversidade, de cursos d'água e do lençol freático, grande parte das veredas está em avançado estado de degradação devido ao desorganizado uso da terra e expansão antrópica no Cerrado (FERREIRA, 2003; Tubelis, 2009). O elevado grau de assoreamento resultante do desmatamento e o pisoteio pelo gado causam compactação do solo, o que consequentemente diminui a taxa de infiltração da água, rebaixa o lençol freático e reduz a vazão das nascentes (MeIReLles et al., 2004). Isso influencia diretamente a comunidade vegetal, ao permitir a colonização por espécies de maior porte e por outras não adaptadas a solos encharcados, o que muda a estrutura ambiental e a composição da fauna associada (GUIMARÃES et al., 2002; MeIrelles et al., 2004). Esse efeito é maior sobre os anfíbios devido a suas restrições ecológicas e fisiológicas em ambientes carentes de umidade (Colli et al., 2002; SEMLITSCH \& Bodie, 2003). Assim, a degradação de veredas pode resultar em declínio e/ou extinção local de diversas populações.

O Chaco é considerado uma província biogeográfica (Morrone, 2006), com diversas espécies endêmicas de anfíbios e répteis (GALlaRdo, 1979; LeYNAUd \& BUCHER, 1999; Brusquetti \& Lavilla, 2006). No Brasil, a representatividade da região chaquenha está restrita a uma pequena faixa, limitada pelas margens dos rios Paraguai e Apa, no município de Porto Murtinho, sul do estado de Mato Grosso do Sul. As primeiras informações detalhadas da herpetofauna do Chaco brasileiro, caracterizado como "Chaco úmido" (Hueck 1972a,b; Prado et al., 1992; Prado, 1993), foram disponibilizadas por Souza et al. (2010). Essa região é a localidade do primeiro registro no Brasil de Melanophryniscus klappenbachi (AMARAL et al., 2012) e Lepidobatrachus asper (SUGAI et al., 2013), conforme previsto por STRÜSSMANN et al. (2011), o que reforça a importância dessa fitofisionomia para a biodiversidade brasileira. Todavia, a região sofre com desmatamentos e queimadas devido à substituição de florestas por gramíneas para a implantação de pecuária (SILVA et al., 2008).

Quanto às macrorregiões consideradas, foram registradas 56 espécies para a planície de inundação (majoritariamente representada pelo Pantanal) e 92 para o planalto, sendo que 51 delas são compartilhadas pelas duas macrorregiões, 41 são espécies exclusivas do planalto e apenas cinco são exclusivas da planície (Tab. I). Estudos 
Tab. I. Espécies de anfíbios registradas para o estado de Mato Grosso do Sul. As espécies são associadas às quatro principais formações vegetais consideradas no estudo (CE, cerrado sensu lato; CH, Chaco; FE, floresta estacional; V, veredas; ver Metodologia), às duas macrorregiões (PI, planície de inundação; PL, planalto) e ao estado de conservação (CR, criticamente em perigo; EN, em perigo; VU, vulnerável; LC, menor risco; DD, dados insuficientes).

\begin{tabular}{|c|c|c|c|c|c|c|c|c|c|c|c|c|}
\hline Espécies & $\mathrm{CE}$ & $\mathrm{CH}$ & $\mathrm{ME}$ & $\mathrm{V}$ & outro & PI & PL & $\mathrm{CR}$ & $\mathrm{EN}$ & VU & LC & DD \\
\hline \multicolumn{13}{|l|}{ ANURA } \\
\hline \multicolumn{13}{|l|}{ Bufonidae } \\
\hline Melanophryniscus fulvoguttatus (Mertens, 1937) & $\mathrm{X}$ & $\mathrm{X}$ & $\mathrm{X}$ & & $\mathrm{X}$ & $\mathrm{X}$ & $\mathrm{X}$ & & & & $\mathrm{X}$ & \\
\hline Melanophryniscus klappenbachi Prigioni \& Langone, 2000 & & $\mathrm{X}$ & & & & $\mathrm{X}$ & & & & & $\mathrm{X}$ & \\
\hline Rhinella aff. scitula & & & $\mathrm{X}$ & & & & $\mathrm{X}$ & & & & & $\mathrm{X}$ \\
\hline Rhinella azarai (Gallardo, 1965) & $\mathrm{X}$ & & & & & & $\mathrm{X}$ & & & & & $\mathrm{X}$ \\
\hline Rhinella bergi (Céspedez, 2000) & $\mathrm{X}$ & $\mathrm{X}$ & $\mathrm{X}$ & & $\mathrm{X}$ & $\mathrm{X}$ & $\mathrm{X}$ & & & & $\mathrm{X}$ & \\
\hline Rhinella major (Muller \& Helmich, 1936) & $\mathrm{X}$ & $\mathrm{X}$ & & & & $\mathrm{X}$ & $\mathrm{X}$ & & & & & $\mathrm{X}$ \\
\hline Rhinella mirandaribeiroi (Gallardo, 1965) & $\mathrm{X}$ & & & & & & $\mathrm{X}$ & & & & & $\mathrm{X}$ \\
\hline Rhinella ocellata (Günther, 1859) & $\mathrm{X}$ & & $\mathrm{X}$ & & & & $\mathrm{X}$ & & & & $\mathrm{X}$ & \\
\hline Rhinella paraguayensis Ávila, Pansonato \& Strüssmann, 2010 & $\mathrm{X}$ & & & & & $\mathrm{X}$ & & & & & & $\mathrm{X}$ \\
\hline Rhinella rubescens (A. Lutz, 1925) & & & $\mathrm{X}$ & & & & $\mathrm{X}$ & & & & $\mathrm{X}$ & \\
\hline Rhinella schneideri (Werner, 1894) & $\mathrm{X}$ & $\mathrm{X}$ & $\mathrm{X}$ & $\mathrm{X}$ & $\mathrm{X}$ & $\mathrm{X}$ & $\mathrm{X}$ & & & & $\mathrm{X}$ & \\
\hline Rhinella scitula (Caramaschi \& Niemeyer, 2003) & $\mathrm{X}$ & & $\mathrm{X}$ & & & $\mathrm{X}$ & $\mathrm{X}$ & & & & & $\mathrm{X}$ \\
\hline \multicolumn{13}{|l|}{ Ceratophryidae } \\
\hline Ceratophrys cranwelli Barrio, 1980 & & $\mathrm{X}$ & & & & $\mathrm{X}$ & $\mathrm{X}$ & & & & $\mathrm{X}$ & \\
\hline Lepidobatrachus asper (Budgett, 1899) & & $\mathrm{X}$ & & & & $\mathrm{X}$ & & & & & & $\mathrm{X}$ \\
\hline \multicolumn{13}{|l|}{ Craugastoridae } \\
\hline Haddadus binotatus (Spix, 1824) & & & $\mathrm{X}$ & & & & $\mathrm{X}$ & & & & $\mathrm{X}$ & \\
\hline \multicolumn{13}{|l|}{ Odontophrynidae } \\
\hline Odontophrynus americanus (Duméril \& Bibron, 1841) & & $\mathrm{X}$ & $\mathrm{X}$ & & $\mathrm{X}$ & $\mathrm{X}$ & $\mathrm{X}$ & & & & $\mathrm{X}$ & \\
\hline Proceratophrys goyana (Miranda-Ribeiro, 1937) & $\mathrm{X}$ & & & & & & $\mathrm{X}$ & & & & $\mathrm{X}$ & \\
\hline Proceratophrys sp. & $\mathrm{X}$ & & & & & & $\mathrm{X}$ & & & & & \\
\hline \multicolumn{13}{|l|}{ Dendrobatidae } \\
\hline Ameerega braccata (Steindachner, 1864) & $\mathrm{X}$ & & & & & & $\mathrm{X}$ & & & & $\mathrm{X}$ & \\
\hline Ameerega picta (Bibron in Tschudi, 1838) & $\mathrm{X}$ & & $\mathrm{X}$ & & $\mathrm{X}$ & & $\mathrm{X}$ & & & & $\mathrm{X}$ & \\
\hline \multicolumn{13}{|l|}{ Hylidae } \\
\hline Dendropsophus araguaya (Napoli \& Caramaschi, 1998) & $\mathrm{X}$ & & & & & & $\mathrm{X}$ & & & & & $\mathrm{X}$ \\
\hline Dendropsophus cerradensis (Napoli \& Caramaschi, 1998) & $\mathrm{X}$ & & & & & & $\mathrm{X}$ & & & & & $\mathrm{X}$ \\
\hline Dendropsophus cruzi (Pombal \& Bastos, 1998) & & & & $\mathrm{X}$ & & & $\mathrm{X}$ & & & & $\mathrm{X}$ & \\
\hline Dendropsophus elianeae (Napoli \& Caramaschi, 2000) & $\mathrm{X}$ & & $\mathrm{X}$ & $\mathrm{X}$ & $\mathrm{X}$ & $\mathrm{X}$ & $\mathrm{X}$ & & & & $\mathrm{X}$ & \\
\hline Dendropsophus jimi (Napoli \& Caramaschi, 1999) & $\mathrm{X}$ & & & $\mathrm{X}$ & $\mathrm{X}$ & & $\mathrm{X}$ & & & & $\mathrm{X}$ & \\
\hline Dendropsophus melanargyreus (Cope, 1887) & & $\mathrm{X}$ & & & & $\mathrm{X}$ & $\mathrm{X}$ & & & & $\mathrm{X}$ & \\
\hline Dendropsophus minutus (Peters, 1872) & $\mathrm{X}$ & $\mathrm{X}$ & $\mathrm{X}$ & $\mathrm{X}$ & $\mathrm{X}$ & $\mathrm{X}$ & $\mathrm{X}$ & & & & $\mathrm{X}$ & \\
\hline Dendropsophus nanus (Boulenger, 1889) & $\mathrm{X}$ & $\mathrm{X}$ & $\mathrm{X}$ & $\mathrm{X}$ & $\mathrm{X}$ & $\mathrm{X}$ & $\mathrm{X}$ & & & & $\mathrm{X}$ & \\
\hline Dendropsophus rubicundulus (Reinhardt \& Lütken, 1862) & & & & $\mathrm{X}$ & $\mathrm{X}$ & & $\mathrm{X}$ & & & & $\mathrm{X}$ & \\
\hline Dendropsophus sanborni (Schmidt, 1944) & $\mathrm{X}$ & & & $\mathrm{X}$ & $\mathrm{X}$ & $\mathrm{X}$ & $\mathrm{X}$ & & & & $\mathrm{X}$ & \\
\hline Dendropsophus tritaeniatus (Bokermann, 1965) & & & & & & & $\mathrm{X}$ & & & & $\mathrm{X}$ & \\
\hline Hypsiboas aff. geographicus & & & $\mathrm{X}$ & $\mathrm{X}$ & & $\mathrm{X}$ & $\mathrm{X}$ & & & & & $\mathrm{X}$ \\
\hline Hypsiboas albopunctatus (Spix, 1824) & $\mathrm{X}$ & & $\mathrm{X}$ & $\mathrm{X}$ & $\mathrm{X}$ & & $\mathrm{X}$ & & & & $\mathrm{X}$ & \\
\hline Hypsiboas caingua (Carrizo, 1991) & $\mathrm{X}$ & & $\mathrm{X}$ & & $\mathrm{X}$ & & $\mathrm{X}$ & & & & $\mathrm{X}$ & \\
\hline Hypsiboas crepitans (Wied-Neuwied, 1824) & $\mathrm{X}$ & & $\mathrm{X}$ & $\mathrm{X}$ & $\mathrm{X}$ & & $\mathrm{X}$ & & & & $\mathrm{X}$ & \\
\hline Hypsiboas lundii (Burmeister, 1856) & $\mathrm{X}$ & & & & & & $\mathrm{X}$ & & & & $\mathrm{X}$ & \\
\hline Hypsiboas paranaiba Carvalho, Giaretta \& Facure, 2010 & & & & & $\mathrm{X}$ & & $\mathrm{X}$ & & & & & $\mathrm{X}$ \\
\hline Hypsiboas punctatus (Schneider, 1799) & $\mathrm{X}$ & $\mathrm{X}$ & $\mathrm{X}$ & $\mathrm{X}$ & $\mathrm{X}$ & $\mathrm{X}$ & $\mathrm{X}$ & & & & $\mathrm{X}$ & \\
\hline Hypsiboas raniceps Cope, 1862 & $\mathrm{X}$ & $\mathrm{X}$ & $\mathrm{X}$ & $\mathrm{X}$ & $\mathrm{X}$ & $\mathrm{X}$ & $\mathrm{X}$ & & & & $\mathrm{X}$ & \\
\hline Hypsiboas sp. & $\mathrm{X}$ & & & & & & $\mathrm{X}$ & & & & & $\mathrm{X}$ \\
\hline Lysapsus limellum (Cope, 1862) & $\mathrm{X}$ & $\mathrm{X}$ & $\mathrm{X}$ & & $\mathrm{X}$ & $\mathrm{X}$ & $\mathrm{X}$ & & & & $\mathrm{X}$ & \\
\hline Osteocephalus taurinus Steindachner, 1862 & $\mathrm{X}$ & & $\mathrm{X}$ & & & & $\mathrm{X}$ & & & & $\mathrm{X}$ & \\
\hline Phyllomedusa azurea Cope, 1862 & $\mathrm{X}$ & $\mathrm{X}$ & $\mathrm{X}$ & $\mathrm{X}$ & $\mathrm{X}$ & $\mathrm{X}$ & $\mathrm{X}$ & & & & & $\mathrm{X}$ \\
\hline Phyllomedusa boliviana Boulenger, 1902 & & & $\mathrm{X}$ & & & & $\mathrm{X}$ & & & & $\mathrm{X}$ & \\
\hline Phyllomedusa sauvagii Boulenger, 1882 & $\mathrm{X}$ & $\mathrm{X}$ & $\mathrm{X}$ & & $\mathrm{X}$ & $\mathrm{X}$ & $\mathrm{X}$ & & & & $\mathrm{X}$ & \\
\hline Pseudis bolbodactyla A. Lutz, 1925 & & & & & $\mathrm{X}$ & & $\mathrm{X}$ & & & & $\mathrm{X}$ & \\
\hline Pseudis paradoxa (Linnaeus, 1758) & $\mathrm{X}$ & $\mathrm{X}$ & $\mathrm{X}$ & $\mathrm{X}$ & $\mathrm{X}$ & $\mathrm{X}$ & $\mathrm{X}$ & & & & $\mathrm{X}$ & \\
\hline Scinax acuminatus(Cope, 1862) & $\mathrm{X}$ & $\mathrm{X}$ & & & $\mathrm{X}$ & $\mathrm{X}$ & $\mathrm{X}$ & & & & $\mathrm{X}$ & \\
\hline Scinax aff. ruber & $\mathrm{X}$ & & $\mathrm{X}$ & $\mathrm{X}$ & $\mathrm{X}$ & $\mathrm{X}$ & $\mathrm{X}$ & & & & & $\mathrm{X}$ \\
\hline Scinax constrictus Lima, Bastos \& Giaretta, 2004 & $\mathrm{X}$ & & $\mathrm{X}$ & $\mathrm{X}$ & & $\mathrm{X}$ & $\mathrm{X}$ & & & & $\mathrm{X}$ & \\
\hline Scinax fuscomarginatus (A. Lutz, 1925) & $\mathrm{X}$ & $\mathrm{X}$ & $\mathrm{X}$ & $\mathrm{X}$ & $\mathrm{X}$ & $\mathrm{X}$ & $\mathrm{X}$ & & & & $\mathrm{X}$ & \\
\hline Scinax fuscovarius (A. Lutz, 1925) & $\mathrm{X}$ & $\mathrm{X}$ & $\mathrm{X}$ & $\mathrm{X}$ & $\mathrm{X}$ & $\mathrm{X}$ & $\mathrm{X}$ & & & & $\mathrm{X}$ & \\
\hline Scinax nasicus (Cope, 1862) & $\mathrm{X}$ & $\mathrm{X}$ & $\mathrm{X}$ & $\mathrm{X}$ & $\mathrm{X}$ & $\mathrm{X}$ & $\mathrm{X}$ & & & & $\mathrm{X}$ & \\
\hline Scinax ruber (Laurenti, 1768) & $\mathrm{X}$ & & & & & $\mathrm{X}$ & $\mathrm{X}$ & & & & $\mathrm{X}$ & \\
\hline Scinax similis (Cochran, 1952) & $\mathrm{X}$ & & & $\mathrm{X}$ & & & $\mathrm{X}$ & & & & $\mathrm{X}$ & \\
\hline Scinax squalirostris (A. Lutz, 1925) & & $\mathrm{X}$ & & $\mathrm{X}$ & $\mathrm{X}$ & & $\mathrm{X}$ & & & & $\mathrm{X}$ & \\
\hline
\end{tabular}


Tab. I. Cont.

\begin{tabular}{|c|c|c|c|c|c|c|c|c|c|c|c|c|}
\hline Espécies & $\mathrm{CE}$ & $\mathrm{CH}$ & ME & $\mathrm{V}$ & outro & PI & PL & $\mathrm{CR}$ & EN & VU & $\mathrm{LC}$ & $\mathrm{DD}$ \\
\hline Scinax x-signatus (Spix, 1824) & & & $\mathrm{X}$ & & & & $\mathrm{X}$ & & & & $\mathrm{X}$ & \\
\hline Trachycephalus typhonius (Linnaeus, 1758) & $\mathrm{X}$ & $\mathrm{X}$ & $\mathrm{X}$ & $\mathrm{X}$ & $\mathrm{X}$ & $\mathrm{X}$ & $\mathrm{X}$ & & & & $\mathrm{X}$ & \\
\hline \multicolumn{13}{|l|}{ Leptodactylidae } \\
\hline Adenomera diptyx Boettger, 1885 & $\mathrm{X}$ & $\mathrm{X}$ & $\mathrm{X}$ & & $\mathrm{X}$ & $\mathrm{X}$ & & & & & $\mathrm{X}$ & \\
\hline Adenomera hylaedactyla (Cope, 1868$)$ & $\mathrm{X}$ & & $\mathrm{X}$ & $\mathrm{X}$ & $\mathrm{X}$ & & $\mathrm{X}$ & & & & $\mathrm{X}$ & \\
\hline Adenomera martinezi (Bokermann, 1956) & $\mathrm{X}$ & & & & & & $\mathrm{X}$ & & & & $\mathrm{X}$ & \\
\hline Adenomera sp. & & & $\mathrm{X}$ & & & & $\mathrm{X}$ & & & & & $\mathrm{X}$ \\
\hline Leptodactylus bufonius Boulenger, 1894 & & $\mathrm{X}$ & & & & $\mathrm{X}$ & $\mathrm{X}$ & & & & $\mathrm{X}$ & \\
\hline Leptodactylus chaquensis Cei, 1950 & $\mathrm{X}$ & $\mathrm{X}$ & $\mathrm{X}$ & $\mathrm{X}$ & $\mathrm{X}$ & $\mathrm{X}$ & $\mathrm{X}$ & & & & $\mathrm{X}$ & \\
\hline Leptodactylus elenae Heyer, 1978 & $\mathrm{X}$ & $\mathrm{X}$ & $\mathrm{X}$ & & $\mathrm{X}$ & $\mathrm{X}$ & $\mathrm{X}$ & & & & $\mathrm{X}$ & \\
\hline Leptodactylus furnarius Sazima \& Bokermann, 1978 & & & & $\mathrm{X}$ & $\mathrm{X}$ & & $\mathrm{X}$ & & & & $\mathrm{X}$ & \\
\hline Leptodactylus fuscus (Schneider, 1799) & $\mathrm{X}$ & $\mathrm{X}$ & $\mathrm{X}$ & $\mathrm{X}$ & $\mathrm{X}$ & $\mathrm{X}$ & $\mathrm{X}$ & & & & $\mathrm{X}$ & \\
\hline Leptodactylus gracilis (Duméril \& Bibron, 1841) & $\mathrm{X}$ & $\mathrm{X}$ & & & $\mathrm{X}$ & & $\mathrm{X}$ & & & & $\mathrm{X}$ & \\
\hline Leptodactylus labyrinthicus (Spix, 1824) & $\mathrm{X}$ & $\mathrm{X}$ & $\mathrm{X}$ & $\mathrm{X}$ & $\mathrm{X}$ & $\mathrm{X}$ & $\mathrm{X}$ & & & & $\mathrm{X}$ & \\
\hline Leptodactylus latrans (Steffen, 1815) & $\mathrm{X}$ & & $\mathrm{X}$ & $\mathrm{X}$ & $\mathrm{X}$ & $\mathrm{X}$ & $\mathrm{X}$ & & & & $\mathrm{X}$ & \\
\hline Leptodactylus mystaceus (Spix, 1824) & $\mathrm{X}$ & & $\mathrm{X}$ & & & & $\mathrm{X}$ & & & & $\mathrm{X}$ & \\
\hline Leptodactylus mystacinus (Burmeister, 1861) & $\mathrm{X}$ & & $\mathrm{X}$ & $\mathrm{X}$ & $\mathrm{X}$ & $\mathrm{X}$ & $\mathrm{X}$ & & & & $\mathrm{X}$ & \\
\hline Leptodactylus podicipinus (Cope, 1862) & $\mathrm{X}$ & $\mathrm{X}$ & $\mathrm{X}$ & $\mathrm{X}$ & $\mathrm{X}$ & $\mathrm{X}$ & $\mathrm{X}$ & & & & $\mathrm{X}$ & \\
\hline Leptodactylus sertanejo Giaretta \& Costa, 2007 & & & & $\mathrm{X}$ & $\mathrm{X}$ & & $\mathrm{X}$ & & & & $\mathrm{X}$ & \\
\hline Leptodactylus syphax Bokermann, 1969 & $\mathrm{X}$ & & $\mathrm{X}$ & $\mathrm{X}$ & $\mathrm{X}$ & $\mathrm{X}$ & $\mathrm{X}$ & & & & $\mathrm{X}$ & \\
\hline Physalaemus albonotatus (Steindachner, 1864) & $\mathrm{X}$ & $\mathrm{X}$ & $\mathrm{X}$ & $\mathrm{X}$ & $\mathrm{X}$ & $\mathrm{X}$ & $\mathrm{X}$ & & & & $\mathrm{X}$ & \\
\hline Physalaemus biligonigerus (Cope, 1861) & $\mathrm{X}$ & & & $\mathrm{X}$ & $\mathrm{X}$ & $\mathrm{X}$ & $\mathrm{X}$ & & & & $\mathrm{X}$ & \\
\hline Physalaemus centralis Bokermann, 1962 & $\mathrm{X}$ & & $\mathrm{X}$ & $\mathrm{X}$ & $\mathrm{X}$ & $\mathrm{X}$ & $\mathrm{X}$ & & & & $\mathrm{X}$ & \\
\hline Physalaemus cuvieri Fitzinger, 1826 & $\mathrm{X}$ & $\mathrm{X}$ & $\mathrm{X}$ & $\mathrm{X}$ & $\mathrm{X}$ & $\mathrm{X}$ & $\mathrm{X}$ & & & & $\mathrm{X}$ & \\
\hline Physalaemus marmoratus (Reinhardt \& Lütken, 1862) & $\mathrm{X}$ & & $\mathrm{X}$ & & & $\mathrm{X}$ & $\mathrm{X}$ & & & & $\mathrm{X}$ & \\
\hline Physalaemus nattereri Steindachner, 1863 & $\mathrm{X}$ & $\mathrm{X}$ & $\mathrm{X}$ & $\mathrm{X}$ & $\mathrm{X}$ & $\mathrm{X}$ & $\mathrm{X}$ & & & & $\mathrm{X}$ & \\
\hline Pseudopaludicola boliviana Parker, 1927 & $\mathrm{X}$ & & & & & $\mathrm{X}$ & $\mathrm{X}$ & & & & $\mathrm{X}$ & \\
\hline Pseudopaludicola falcipes (Hensel, 1867) & $\mathrm{X}$ & & & & & $\mathrm{X}$ & $\mathrm{X}$ & & & & $\mathrm{X}$ & \\
\hline Pseudopaludicola mystacalis (Cope, 1887) & $\mathrm{X}$ & & $\mathrm{X}$ & $\mathrm{X}$ & $\mathrm{X}$ & $\mathrm{X}$ & $\mathrm{X}$ & & & & $\mathrm{X}$ & \\
\hline Pseudopaludicola saltica (Cope, 1887) & $\mathrm{X}$ & & $\mathrm{X}$ & $\mathrm{X}$ & $\mathrm{X}$ & $\mathrm{X}$ & $\mathrm{X}$ & & & & $\mathrm{X}$ & \\
\hline Pseudopaludicola ternetzi Miranda-Ribeiro, 1937 & & & & $\mathrm{X}$ & & & $\mathrm{X}$ & & & & $\mathrm{X}$ & \\
\hline Pseudopaludicola sp. & & & & $\mathrm{X}$ & $\mathrm{X}$ & & $\mathrm{X}$ & & & & & $\mathrm{X}$ \\
\hline \multicolumn{13}{|l|}{ Microhylidae } \\
\hline Chiasmocleis albopunctata (Boettger, 1885) & $\mathrm{X}$ & $\mathrm{X}$ & $\mathrm{X}$ & $\mathrm{X}$ & & $\mathrm{X}$ & $\mathrm{X}$ & & & & $\mathrm{X}$ & \\
\hline Chiasmocleis mehelyi Caramaschi \& Cruz, 1997 & $\mathrm{X}$ & & & & $\mathrm{X}$ & $\mathrm{X}$ & & & & & & $\mathrm{X}$ \\
\hline Dermatonotu muelleri (Boettger, 1885) & $\mathrm{X}$ & $\mathrm{X}$ & $\mathrm{X}$ & $\mathrm{X}$ & $\mathrm{X}$ & $\mathrm{X}$ & $\mathrm{X}$ & & & & $\mathrm{X}$ & \\
\hline Elachistocleis bicolor (Valenciennes in Guérin-Menéville, 1838) & $\mathrm{X}$ & $\mathrm{X}$ & $\mathrm{X}$ & $\mathrm{X}$ & $\mathrm{X}$ & $\mathrm{X}$ & $\mathrm{X}$ & & & & $\mathrm{X}$ & \\
\hline Elachistocleis cesarii (Miranda Ribeiro (1920) & $\mathrm{X}$ & & $\mathrm{X}$ & $\mathrm{X}$ & & $\mathrm{X}$ & $\mathrm{X}$ & & & & & $\mathrm{X}$ \\
\hline Elachistocleis matogrosso Caramaschi, 2010 & $\mathrm{X}$ & & $\mathrm{X}$ & & & $\mathrm{X}$ & $\mathrm{X}$ & & & & & $\mathrm{X}$ \\
\hline Elachistocleis sp. & & & & $\mathrm{X}$ & $\mathrm{X}$ & & $\mathrm{X}$ & & & & & $\mathrm{X}$ \\
\hline \multicolumn{13}{|l|}{ Strabomantidae } \\
\hline Oreobates crepitans (Bokermann, 1965) & $\mathrm{X}$ & & $\mathrm{X}$ & & & & $\mathrm{X}$ & & & & & $\mathrm{X}$ \\
\hline Pristimantis dundeei (Heyer \& Muñoz, 1999) & & & $\mathrm{X}$ & & & & $\mathrm{X}$ & & & & & $\mathrm{X}$ \\
\hline \multicolumn{13}{|l|}{ GYMNOPHIONA } \\
\hline \multicolumn{13}{|l|}{ Siphonopidae } \\
\hline Siphonops paulensis Boettger, 1892 & $\mathrm{X}$ & & $\mathrm{X}$ & & & & $\mathrm{X}$ & & & & $\mathrm{X}$ & \\
\hline
\end{tabular}

anteriores consideraram a riqueza de anuros no Pantanal em Mato Grosso do Sul como algo em torno de 45 espécies (UetanABARo et al., 2008; STRÜSSMANn et al., 2011). Apesar de o Pantanal ser um ambiente com predomínio de áreas alagadas, o que configura uma característica ambiental importante para os anfíbios, é uma das regiões brasileiras com maior escassez de informações. A lista de espécies deste trabalho reuniu informações decorrentes de registros recentes ou que ainda não haviam sido publicados, resultando, portanto, em maior número de espécies, o que é natural em contribuições envolvendo inventários.

O ciclo de cheia e seca no Pantanal, com seus processos ecológicos resultantes do pulso de inundação, é bastante variável em termos de recorrência e duração, o que é definido não só pelas chuvas, mas também pelo relevo, incluindo o planalto do entorno (GoNÇALVES et al., 2011;
MerCANTE et al., 2011). Dessa maneira, períodos de seca que se prolonguem por vários meses em anos consecutivos podem limitar a disponibilidade de água em determinados períodos, dificultando assim a presença de animais dependentes de ambientes úmidos e alagados como os anfíbios. Além disso, sabe-se que a planície é (e será) influenciada pelo uso e gestão das áreas dos rios que drenam para o Pantanal, localizadas no planalto e mesmo nos estados limítrofes ao Mato Grosso do Sul (ver discussão em AlHo \& SABINo, 2011).

$\mathrm{O}$ anuro Lepidobatrachus asper encontra-se sob ameaça no Paraguai (Quase Ameaçado - NT) devido à sua área de distribuição ser muito restrita (aproximadamente 20.000 $\mathrm{km}^{2}$ ) e suas populações estarem em declínio, provavelmente devido a incêndios e conversão de áreas naturais em pastagem (Aquino et al., 2004). No Brasil, a espécie é conhecida por um único registro (SUGAI et al., 2013), em região severamente 
fragmentada e associada a um domínio fitogeográfico (Chaco) com pequena representatividade no país. Entretanto, não há informações suficientes acerca de flutuações extremas na extensão de ocorrência, área de ocupação ou qualidade do hábitat para averiguar o atual estado de conservação dessa espécie no Brasil. Assim, L. asper foi considerada dentro do critério de "dados insuficientes" (DD). Algumas espécies ainda não identificadas e que provavelmente são novas para a ciência, pertencentes aos gêneros Phyllomedusa, Elachistocleis e Adenomera podem estar ameaçadas de extinção por ocorrerem em áreas muito restritas (regiões da Serra de Maracaju em Aquidauana e do Maciço do Urucum, em Corumbá), sujeitas à degradação ambiental decorrente da expansão agrícola, pecuária e da extração de minério de ferro e manganês.

Todas as fitofisionomias consideradas nesse estudo sofrem impactos de diversas naturezas antrópicas, incluindo fogo, drenagem, assoreamento, implementação de pequenas centrais hidrelétricas, substituição de pastagens nativas e desmatamento para implementação de pastagens, monoculturas (e.g., cana-de-açúcar, eucalipto) e agricultura (MACHADO et al., 2004; HARRIs et al., 2005; AlHO \& SABINO, 2011), ameaçando, assim, a biodiversidade regional. Esses fatores apontam para clara necessidade de maiores esforços na busca de informações que associem ecologia, manejo e conservação em diferentes escalas espaciais e temporais.

$\mathrm{O}$ estado tem potencial para abrigar mais espécies. As regiões mais amostradas em Mato Grosso do Sul são o entorno do Parque Nacional da Serra da Bodoquena e algumas áreas do Pantanal (Figs 1, 2). Há lacunas de amostragens por todo o estado, principalmente nas regiões norte, leste/ sudeste e parte do oeste/sudoeste (Figura 3). É possível destacar sete áreas para futuras amostragens (Fig. 1): Maciço do rio Apa (1), Serra de Maracaju (3a, 3b e 3c), grande parte do Chaco brasileiro (4), leque do rio Taquari (7), planalto do Taquari-Itiquira (8), escarpas do planalto Central (9) e áreas úmidas/florestas estacionais da planície do rio Paraná (10). Essas áreas estão situadas em regiões com pouco ou nenhum registro no estado, e foram apontadas como foco para inventários por possuírem alta heterogeneidade de relevo (Fig. 1), serem regiões alagáveis (Fig. 2) e/ou apresentarem considerável extensão de vegetação nativa. Destaca-se, também, que nessas regiões apontadas existem Unidades de Conservação que praticamente não foram amostradas (Fig. 2), incluindo, por exemplo, o Monumento Natural Serra Bom Jardim e o Parque Estadual Nascentes do Taquari (Fig. 2; pontos 10 e 12 respectivamente), que fazem parte do corredor Cerrado-Pantanal ou o Parque Estadual das Várzeas do Rio Ivinhema, o Parque Natural Municipal de Naviraí e o Parque Nacional de Ilha Grande (Fig. 2; pontos
15,16 e 17), que representam importantes fragmentos de Floresta Estacional. Embora um dos objetivos das Unidades de Conservação seja proporcionar meios e incentivos para a pesquisa científica (SNUC, 2006), o atraso na regularização fundiária e efetiva implantação dessas Unidades impedem que essa prática.

Há carência de recursos humanos qualificados atuando diretamente nas instituições de ensino e pesquisa do estado, o que limita a construção e maior divulgação do conhecimento. Por exemplo, uma busca no diretório do CNPq identificou apenas dois Grupos de Pesquisa envolvidos diretamente com o estudo de anfíbios em Mato Grosso do Sul. Ambos os grupos estão vinculados à UFMS, em Campo Grande (História Natural de Anfíbios e Répteis coordenado por Vanda Lúcia Ferreira e História Natural e Ecologia de Vertebrados em Ambientes Naturais e Antrópicos - coordenado por Franco Leandro de Souza). Entretanto, ambos os grupos interagem com pesquisadores de outras instituições e de outras áreas do conhecimento. As coleções científicas do estado são escassas e carecem de recursos para seu bom funcionamento, o que demanda mais tempo para o tombamento de exemplares e, consequentemente, retarda a disponibilidade dos dados. Vale destacar, entretanto, que um aporte recente de recursos financeiro através do programa estadual Biota-MS possibilitou a permanência de bolsistas nas coleções científicas, o que tem resultado em significativo incremento do número de animais tombados.

Amostragens da fauna e flora, seja para montar listas de espécies ou para testar teorias ecológicas, são extremamente importantes em regiões pouco conhecidas e com grande diversidade de ambientes, como é o caso do Mato Grosso do Sul. Além de preencher lacunas de conhecimento sobre a diversidade e padrão de distribuição das espécies de anfíbios no estado, também propiciam material para trabalhos que buscam resolver problemas de identificação e classificação das espécies. Essas revisões taxonômicas são importantes, não só para um trabalho de sistemática consistente, mas também para a aplicação de conceitos de Biologia da Conservação, já que as espécies são as unidades básicas na estimativa de diversos parâmetros de biodiversidade (AleIXo, 2009). Assim, é necessário maior esforço de coleta incluindo também girinos, amostras de tecidos para análises moleculares e registros acústicos de espécies que ocorrem em Mato Grosso do Sul. Estes tipos de materiais biológicos ainda estão pouco disponíveis aos pesquisadores e raramente são mencionados em revisões taxonômicas (NARVAes \& Rodrigues, 2009; CARAMASCHI, 2010; GARDA et al., 2010), o que pode levar à subestimação da riqueza e omissão de espécies novas e/ ou ameaçadas. 


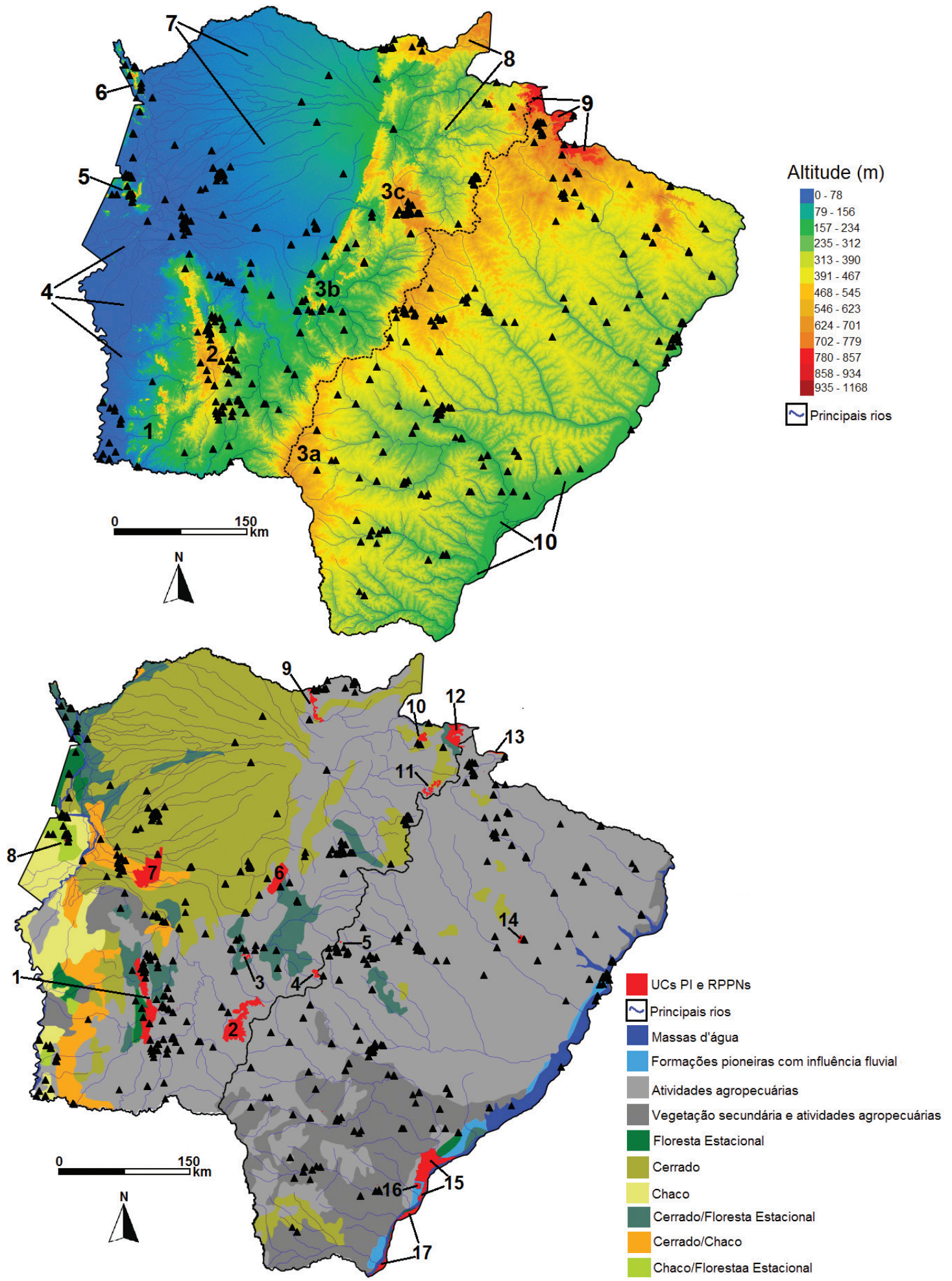

Figs 1, 2. Mapas do estado de Mato Grosso do Sul indicando a localização dos registros de anfíbios (triângulos pretos). A linha tracejada que cruza o estado indica a divisa entre as bacias dos rios Paraguai e Paraná (Fonte: ANA 2012). Fig. 1. Mapa de altitude (os números indicam algumas áreas de interesse citadas no texto): 1, Maciço do rio Apa; 2, Planalto da Bodoquena; 3, Planalto de Maracaju-Campo Grande; 4, Áreas alagáveis na planície do rio Paraguai com predomínio de Chaco (ver Fig. 2); 5, Maciço do Urucum; 6, Serra do Amolar; 7, Leque do rio Taquari; 8, Planalto do Taquari-Itiquira; 9, Escarpas do Planalto Central; 10, Áreas alagáveis na planície do rio Paraná. Fig. 2. Mapa de vegetação segundo a classificação proposta nesse trabalho (ver Metodologia; as veredas são muito pequenas para aparecer na escala do mapa) e Unidades de Conservação de Proteção Integral (destacadas em vermelho): 1, Parque Nacional da Serra da Bodoquena; 2, Monumento Natural Serra de Maracaju; 3, Monumento Natural Morrarias de Anastácio; 4, Monumento Natural Serra de Terenos; 5, Parque Estadual Matas do Segredo e Parque Estadual do Prosa; 6, Monumento Natural Serra de MaracajuCorguinho; 7, Parque Estadual do Pantanal do Rio Negro; 8, Parque Natural Municipal de Piraputangas; 9, Parque Estadual Serra de Sonora e Monumento Natural Serra do Pantanal; 10, Monumento Natural Serra Bom Jardim e Parque Natural Municipal Templo dos Pilares; 11, Monumento Natura Serra de Figueirão; 12, Parque Estadual das Nascentes do Taquari; 13, Parque Nacional das Emas; 14, Parque Natural Municipal Pombo; 15, Parque Estadual das Várzeas do Rio Ivinhema; 16, Parque Natural Municipal de Naviraí; 17, Parque Nacional de Ilha Grande. 


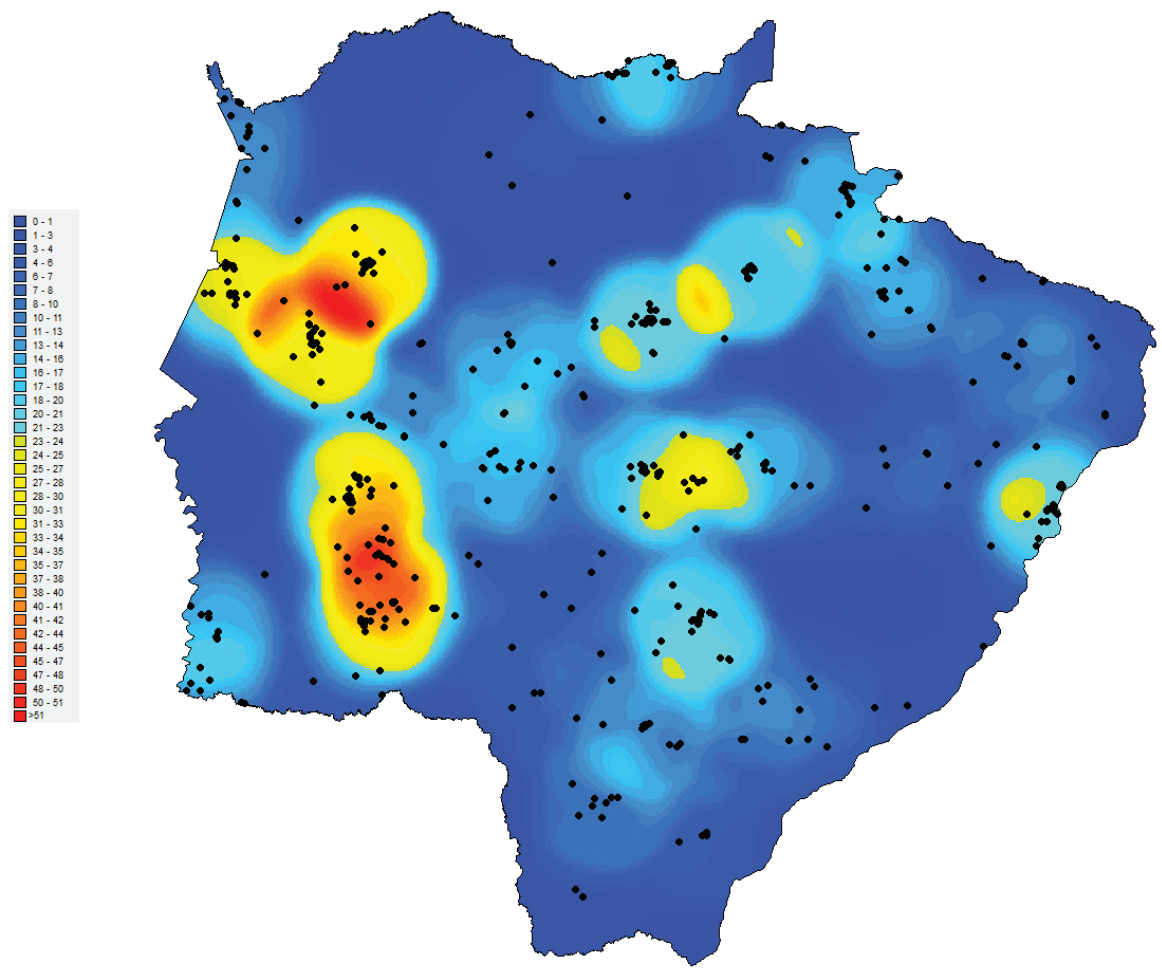

Fig. 3. Mapa do estado de Mato Grosso do Sul indicando a localização dos registros de anfíbios (pontos pretos) e as áreas de maior (vermelho) e menor (azul) conhecimento, evidenciando as lacunas de amostragens. As cores indicam a amplitude do número de amostras.

Agradecimentos. A Fundação de Apoio ao Desenvolvimento do Ensino, Ciências e Tecnologia do Estado de Mato Grosso do Sul (Fundect) e a Superintendência de Ciências e Tecnologia do Estado de Mato Grosso do Sul (Sucitec/MS) pelo convite de participação neste fascículo especial da Iheringia, Série Zoologia e o suporte financeiro para sua publicação. Renato Bérnils, Guarino R. Colli, Reuber A. Brandão, Rogério P. Bastos, Célio F.B. Haddad e Paula H. Valdujo gentilmente cederam dados de campo e auxiliaram na identificação de várias espécies; Universidade Federal de Mato Grosso do Sul, Conselho Nacional de Desenvolvimento Científico e Tecnológico, Fundação de Apoio ao Desenvolvimento do Ensino, Ciência e Tecnologia do Estado de Mato Grosso do Sul e Instituto Homem Pantaneiro pelo apoio logístico em diversas ocasiões. FLS, VLF, CS e DJR são pesquisadores do CNPq. C.P.A. Prado é grata ao CNPq (Processo 471106/2010-0) e a FAPESP (Processo 2009/12013-4) pelo auxílio financeiro.

\section{REFERÊNCIAS BIBLIOGRÁFICAS}

ADÁmoli, J. 1982. O Pantanal e suas relações fitogeográficas com os cerrados: discussão sobre o conceito de complexo do Pantanal. In: Anais do $32^{\circ}$ Congresso Nacional da Sociedade Botânica do Brasil. Teresina, Sociedade Brasileira de Botânica, p.109-119.

Aleixo, A. 2009. Conceitos de espécie e suas implicações para a conservação. Megadiversidade 5(1-2):87-95.

Alho, C. J. R. \& SABINo, J. 2011. A conservation agenda for the Pantanal's biodiversity. Brazilian Journal of Biology 71(suppl.1):327-335.

Almeida, S. C.; Maffei, F.; Rolim, D. C.; Ubaid, F. K. \& Jim, J. 2008. Amphibia, Anura, Hylidae, Sphaenorhynchus caramaschii: Distribution extension in state of São Paulo, Brazil. Check List 4(4):439-441.

Amaral, I. B.; Landgref-Filho , P.; Souza, F. L. \& Uetanabaro, M. 2012. Melanophryniscus klappenbachi (Klappenbach's Red -bellied Toad). Herpetological Review 43(1):98.

АмPHIBIAWев. 2012. Information on amphibian biology and conservation. Disponível em <http://amphibiaweb.org/. > Acessado em 11.09.2012.

ANA - Agência Nacional de Águas. Sistema de Informações Hidrológicas. Disponível em <http://hidroweb.ana.gov.br/>. Acessado em 08.2012.
Andrade, S. P. \& VAZ-Silva, W. 2012. First state record and distribution extension of Pleurodema diplolister (Peters 1870) (Anura: Leiuperidae) from state of Goiás, Brazil. Check List 8(1):149-151.

Aoki, C.; Landgref-Filho, P.; Sousa, D. L. H.; Oda, F. H.; Gamarra, R. M. \& SouZA, F. L. 2010. Hypsiboas caingua (Striped Treefrog). Herpetological Review 41(4):507.

Aoki, C.; Landgref-Filho, P.; Oda, F. H. \& Gamarra, R. M. 2011. Rhinella rubescens. Herpetological Review 42(1):108.

Aquino, L.; Tada, I. \& Faivovich, J. 2004. Lepidobatrachus asper. In: IUCN 2012. IUCN Red List of Threatened Species. Version 2012.1. Disponível em $<\mathrm{http}: / /$ www.iucnredlist.org $>$. Acessado em 25.09.2012.

Ávila, R. W. \& Ferreira, V. L. 2004. Riqueza e densidade de vocalizações de anuros (Amphibia) em uma área urbana de Corumbá, Mato Grosso do Sul. Revista Brasileira de Zoologia 21(4):887-892.

Ávila, R. W.; Pansonato, A. \& Strüssmann, C. 2010. A new species of the Rhinella margaritifera group (Anura: Bufonidae) from Brazilian Pantanal. Zootaxa 2339:57-68.

Batalha, M. A. 2011. O cerrado não é um bioma. Biota Neotropica 11(1):21-24.

Becker, C. G.; Loyola, R. D.; Haddad, C. F. B. \& Zamudio, K. R. 2010. Integrating species life-history traits and patterns of deforestation in amphibian conservation planning. Diversity and Distributions 16(1):10-19.

Blackburn, D. C. \& WaKe, D. B. 2011. Class Amphibia Gray, 1825. In Animal biodiversity: an outline of higher-level classification and survey of taxonomic richness (Z.Q. Zhang, ed.). Zootaxa 3148:38-54.

Brusquetti, F. \& Lavilla, E. O. 2006. Lista comentada de los anfibios de Paraguay. Cuadernos de herpetología 20(2):3-79.

CARAMASCHI, U. 2010. Notes on the taxonomic status of Elachistocleis ovalis (Schneider, 1799) and description of five new species of Elachistocleis Parker, 1927 (Amphibia, Anura, Microhylidae). Boletim do Museu Nacional, Nova Série Zoologia 527:1-30.

Colli, G. R.; Bastos, R. P. \& AraúJo, A. F. B. 2002. The character e dynamics of the Cerrado Herpetofauna. In: Oliveira, P. S. \& MARQuis, R. J. eds. The Cerrados of Brazil: Ecology and Natural History of a Neotropical Savanna. NewYork, Columbia University Press, p.223-241. 
Diniz-Filho, J. A. F.; Bini, L. M.; Pinto, M. P.; Rangel, T. F. L. V. B.; Carvalho, P.; Vieira, S. L. \& Bastos, R. P. 2007. Conservation biogeography of anurans in Brazilian Cerrado. Biodiversity and Conservation 16(4):997-1008.

Diniz-Filho, J. A. F.; Bini, L. M.; Vieira, C. M.; Blamires, D.; Terrible, L.; Bastos, R.; De Oliveira, G. \& Barreto, B. 2008. Spatial patterns of terrestrial vertebrate species richness in the Brazilian Cerrado. Zoological Studies 47(2):146-157.

Faivovich, J.; Haddad, C. F. B.; Garcia, P. C. A.; Frost, D. R.; Campbell, J. A. \& WheELler, W. C. 2005. Systematic review of the frog family Hylidae, with special reference to Hylinae: phylogenetic analysis e taxonomic revision. Bulletin of the American Museum of Natural History 294:1-240.

Ferreira, I. M. 2003. Consequências da ocupação do Cerrado: morte do subsistema de Veredas. Espaço em Revista 4(1):18-23.

Filho, W. S.; Karmani, I. \& Boggiani, P. C. 2004. Paisagens cársticas da Serra da Bodoquena (MS). In: Mantesso-Neto, V.; Bartorelli, A.; Carneiro, C. D. R. \& Brito-Neves, B. B. eds. Geologia do Continente Sul-Americano: Evolução da Obra de Fernando Flávio Marques de Almeida. São Paulo, Beca, p.423-433.

Gallardo, J. M. 1979. Composición, distribución y origem de la herpetofauna chaquena. In: Duellman, W. E. ed. The South American herpetofauna: its origin, evolution, and dispersal. Monographs of the Museum of Natural History of the University of Kansas 7:299-307.

Garda, A. A.; Santana, D. J. \& SÃo-Pedro, V. D. A. 2010. Taxonomic characterization of Paradoxical frogs (Anura, Hylidae, Pseudae): geographic distribution, external morphology, and morphometry. Zootaxa 2666:1-28.

Godoy, A. M.; Manzano, J. C.; Araújo, L. M. B. \& Silva, J. A. 2009. Contexto geológico e estrutural do Maciço Rio Apa, sul do Cráton Amazônico - MS. Geociências 28(4):485-499.

Gonçalves, H. C.; Mercante, M. A. \& Santos, E. T. 2011. Hydrological cycle. Brazilian Journal of Biology 71(suppl. 1):241-253.

Gordo, M. \& CAmpos, Z. 2003. Listagem de anuros da Estação Ecológica Nhumirim e arredores, Pantanal Sul. Embrapa Pantanal. Documentos 58:1-21.

Gordo, M. \& Campos, Z. M. S. 2005. Anuros das serras de entorno do Pantanal Sul. Embrapa Pantanal. Documentos 78:1-21.

Guimarẽes, A. J. M.; AraúJo, G. M. \& CorrêA, G. F. 2002. Estrutura fitossociológica em área natural e antropizada de uma vereda em Uberlândia, MG. Acta Botanica Brasilica 16(3):317-329.

Harris, M. B.; Tomas, W. M.; Mourão, G.; Silva, C. J.; Guimarães, E.; SonodA, F. \& FACHIM, E. 2005. Desafios para proteger o Pantanal brasileiro: ameaças e iniciativas em conservação. Megadiversidade 1(1):156-164.

Hedges, S. B.; Duellman, W. E. \& Heinicke, M. P. 2008. New World direct-developing frogs (Anura: Terrarana): molecular phylogeny, classification, biogeography, and conservation. Zootaxa 1737:1-182.

Hijmans, R. J.; Guarino, L. \& Rojas, E. 2002. DIVA-GIS. A geographic information system for the analysis of biodiversity data. Manual. Lima, International Potato Center. 73p.

Hueck, K. 1972a. As florestas da América do Sul: ecologia, composição e importância econômica. São Paulo, Polígono, Ed. UnB. 466p.

Hueck, K. 1972b. Mapa de Vegetación de América del Sur. Stuttgart, Gustav Fischer Verlag.

IUCN. 2001. IUCN Red List Categories and Criteria: version 3.1. IUCN, Species Survival Commission. Disponível em $<\mathrm{http}$ ://www.iucnredlist. org/technical-documents/categories-and-criteria/2001-categoriescriteria>. Acessado em 18.09.2012.

Klink, C. A. \& Machado, R. B. 2005. Conservation of the Brazilian Cerrado. Conservation Biology 19(3):707-713

Kolenc, F.; Baldo, D.; Borteiro, C.; Marangoni, F.; Ferraro, D. P. \& Faivovich, J. 2011. The identity of Eupemphix fuscomaculatus Steindachner, 1864 (Amphibia: Anura). Copeia 2011(4):513-522.

LANDGref-Filho, P.; GodoI, M. N. \& Souza, F. L. 2012. Dendropsophus sanborni (Sanborn's Treefrog). Herpetological Review 43(1):97.

Lavilla, E. O.; Langone, J. A.; Padial, J. A. \& De Sá, R. O. 2010. The identity of the crackling, luminescent frog of Suriname (Rana typhonia Linnaeus, 1758) (Amphibia, Anura). Zootaxa 2671:17-30.

Leynaud, G. C. \& Bucher, E. H. 1999. La fauna de serpientes del Chaco Sudamaricano: divsersidad, distribución geográfica y estado de conservación. Academia Nacional de Ciencias de Córdoba, Miscelánea 98:1-46.

Loyola, R. D.; Becker, C. G.; Kubota, U.; Haddad, C. F. B.; Fonseca, C. R. \& Lewinsohn, T. M. 2008. Hung out to dry: choice of priority ecoregions for conserving threatened Neotropical anurans depends on life-history traits. Plos One 3(5):e2120. doi:10.1371/journal. pone. 0002120

Machado, R. B.; Ramos Neto, M. B.; Pereira, P. G. P.; Caldas, E. F.; Gonçalves, D. A.; Santos, N. S.; TABor, K. \& Steininger, M. 2004. Estimativas de perda da área do Cerrado brasileiro. Relatório técnico não publicado. Brasília, Conservação Internacional.

Meirelles, M. L.; Guimarães, A. J. M.; Oliveira, R. C.; Araújo, G. M. \& RIBEIRo, J. F. 2004. Impactos sobre o estrato herbáceo de áreas úmidas de Cerrado. In: Aguiar, L. M. \& Camargo, A. J. A. eds. Cerrado: Ecologia e Caracterização. Planaltina, Embrapa Cerrados, p. 41-68.

Mercante, M. A.; Rodrigues, S. C. \& Ross, J. L. S. 2011. Geomorphology and habitat diversity in the Pantanal. Brazilian Journal of Biology 71(suppl. 1):233-240.

Miles, L.; Newton, A. C.; Defries, R. S.; Ravilious, C.; May, I.; Blyth, S.; Kapos, V.\& Gordon, J. E. 2006. A global overview of the conservation status of tropical dry forests. Journal of Biogeography 33(3):491-505.

Morrone, J. 2006. Biogeographic areas and transition zones of Latin America and the Caribbean Islands based on panbiogeographic and cladistic analyses of the entomofauna. Annual Review of Entomology 51:467-494.

Moura, M. R.; Fernandes, V. D.; Santana, D. J. \& Feio, R. N. 2010. Amphibia, Anura, Hylidae, Scinax centralis Pombal and Bastos, 1996: Distribution extension, geographic distribution map. Check List 6(1):173-175

Myers, N.; Mittermeier, R. A.; Mittermeier, C. G.; Fonseca, G. A. B. \& Kent, J. 2000. Biodiversity hotspots for conservation priorities. Nature 403:853-858.

Narvaes, P. \& Rodrigues, M. T. 2009. Taxonomic revision of Rhinella granulosa species group (Amphibia, Anura, Bufonidae), with a description of a new species. Arquivos de Zoologia 40(1):1-73.

Nascimento, L. B.; Pimenta, B. V. S.; Cruz, C. A. G. \& Caramaschi, U. 2006. Taxonomic status of Gomphobates marmoratus Reinhardt and Lutken, 1862 "1861" and Eupemphix fuscomaculatus Steindachner, 1864 (Amphibia, Anura, Leptodactylidae). South American Journal of Herpetology 1(3):166-174.

Padial, J. M.; Chaparro, J. C.; Castroviejo-Fisher, S.; Guayasamin, J. M.; Lehr, E.; Delgado, A. J.; Vaira, M.; Teixeira JR., M.; Aguayo, R. \& De LA RIVA, I. 2012. A revision of species diversity in the Neotropical genus Oreobates (Anura: Strabomantidae), with the description of three new species from the Amazonian slopes of the Andes. American Museum Novitates 3752:1-55.

PCBAP. 1997. Projeto Pantanal. Programa Nacional do Meio Ambiente (PNMA). Subcomponente Pantanal. Plano de Conservação da Bacia do Alto Paraguai - PCBAP. Diagnóstico dos Meios Físico e Biótico. Meio Biótico. Brasília, MMA/PNMA. vol.2, t.3, p.1-433.

Pott, A.; Oliveira, A. K. M.; Damasceno-Junior, G. A. \& Silva, J. S. V. 2011. Plant diversity of the Pantanal wetland. Brazilian Journal of Biology 71(suppl.1):265-273.

Prado, D. E. 1993. What is the Gran Chaco vegetation in South America? I. A review. Contribution to the study of flora and vegetation of the Chaco, V. Candollea 48(1):145-172.

Prado, D. E. \& GibBs, P. E. 1993. Patterns of species distributions in the dry seasonal forests of South America. Annals of the Missouri Botanical Garden 80(4):902-927.

Prado, D. E.; Gibbs, P. E.; Pott, A. \& Pott, V. J. 1992. The Chaco-Pantanal transition in southern Mato Grosso, Brazil. In: FurLey, P. A.; Proctor, J. \& Ratter, J. A. eds. Nature and Dynamics of Forest-Savanna Boundaries. London, Chapman and Hall, p.451-470.

Pyron, A. \& Wiens, J. J. 2011. A large-scale phylogeny of Amphibia with over 2,800 species, and a revised classification of extant frogs, salamanders, and caecilians. Molecular Phylogenetics and Evolution 61(2):543-583.

Quantum Gis Development Team. 2012. Quantum GIS Geographic Information System. Open Source Geospatial Foundation Project. Disponível em $<$ http://qgis.osgeo.org $>$. 
Rossa-Feres, D. C.; Sawaya, R. J.; Faivovich, J.; Giovanelli, J. G. R.; Brasileiro, C. A.; Schiesari, L.; Alexandrino, J. \& Haddad, C. F. B. 2011. Amphibians of São Paulo State, Brazil: state-of-art and perspectives. Biota Neotropica 11(1a):1-19.

Segalla, M.V.; Caramaschi, U.; Cruz, C.A.G.; Grant, T.; Haddad, C.F.B.; LANGONE, J.A. \& GARCIA, P.C.A. 2014. Brazilian Amphibians: List of Species. Herpetologia Brasileira 3(2):37-48.

Semlitsch, R. D. \& Bodie, J. R. 2003. Biological criteria for buffer zones around wetlands e riparian habitats for amphibians e reptiles. Conservation Biology 17(5):1219-1228.

Silva, J. F.; Fariñnas, M. R.; Felfili, J. M. \& Klink, C. A. 2006. Spatial heterogeneity, land use and conservation in the Cerrado region of Brazil. Journal of Biogeography 33(3):536-548.

Silva, J. M. C. \& BATES, J. M. 2002. Biogeographic patterns and conservation in the South American Cerrado: a tropical savanna hotspot. BioScience 52(3):225-234

Silva, M. P.; Mauro, R. A.; Abdon, M. \& Silva, J. S. V. 2008. Estado de Conservação do Chaco (Savana Estépica) Brasileiro. In: IX Simpósio Nacional Cerrado. Desafios e estratégias para o equilíbrio entre sociedade, agronegócio e recursos naturais. Brasília, Embrapa Cerrados, p. 1-6.

Silva, R. A.; Martins, I. A. \& Rossa-Feres, D. C. 2011. Environmental heterogeneity: anuran diversity in homogeneous environments. Zoologia 28(5):610-618

Siqueira, C. C.; Vrcibradic, D.; Almeida-Gomes, M.; Borges-Junior, V. N. T.; Almeida- Santos, P.; Almeida- Santos, M.; Ariani, C. V.; Guedes, D. M.; Goyannes- Araújo, P.; Dorigo, T. A.; Van Sluys, M. \& Rocha, C. F. D. 2009. Density and richness of leaf litter frogs (Amphibia: Anura) of an Atlantic Rainforest area in the Serra dos Órgãos, Rio de Janeiro State, Brazil. Zoologia 26(1):97-102.

SNUC. 2006. Sistema Nacional de Unidades de Conservação. Lei N. 9.985 de 18 de julho de 2000, Decreto N. 4.340, de 22 de agosto de 2002 . Brasília, SNUC. $7^{\mathrm{a}}$ ed.

Souza, F. L.; Uetanabaro, M.; Landgref-Filho, P.; Piatti, L. \& Prado, C. P. A. 2010. Herpetofauna, municipality of Porto Murtinho, Chaco region, state of Mato Grosso do Sul, Brazil. Check List 6(3):470-475.

Souza Filho, G. A. \& Lima, A. M. X. 2012. Hypsiboas caingua (Carrizo, 1990) (Amphibia: Anura: Hylidae): Geographic distribution with a new state record in Brazil. Check List 8(4):800-801.

Strüssmann, C.; Prado, C. P. A.; Uetanabaro, M. \& Ferreira, V. L. 2000. Levantamento de anfíbios e répteis de localidades selecionadas na porção sul da planície alagada do Pantanal e Cerrado do entorno, Mato Grosso do Sul, Brasil. In: Willink, P. W.; Chernoff, B.; Alonso, L. E.; Montambault J. R. \& Lourival, R. eds. A Biological assessment of the aquatic ecosystems of the Pantanal, Mato Grosso do Sul, Brazil. Washington, Conservation International. (RAP Bulletin of Biological Assessment, n.18. p.219-223).

Strüssmann, C.; Kawashita-Ribeiro, R. A.; Ferreira, V. L. \& Béda, A. F. 2007. Herpetofauna do Pantanal Brasileiro. In: NAscimento, L. B. \& Oliveira, M. E. eds. Herpetologia no Brasil II. Belo Horizonte, Sociedade Brasileira de Herpetologia. p.66-84.

Strüssmann, C.; Prado, C. P. A.; Ferreira, V. L. \& Kawashita-Ribeiro, R. A. 2011. Diversity, ecology, management and conservation of amphibians and reptiles of the Brazilian Pantanal: a review. In: JUNK, W. J.; Da Silva, C. J.; Nunes da Cunha, C. \& Wantzen, K. M. eds. The Pantanal: Ecology, biodiversity and sustainable management of a large neotropical seasonal wetland. Sofia, Pensoft Publishers, p.495-519.

Sugai, J. L. M. M.; Souza, F. L.; Uetanabaro, M. \& Landgref-Filho, P. 2012. Geographic distribution: Rhinella azarai (Azara's granulated toad). Herpetological Review 43:441.

Sugai, J. L. M. M.; Faggioni, G. P.; Piatti, L.; Lemos, A. A.; Souza, F. L. \& Prado, C. P. A. 2013. Lepidobatrachus asper Budgett, 1899 (Amphibia: Anura: Ceratophryidae): New country record, distribution map and natural history notes. Check List 9(1):133-135.

Toledo, L. F. \& BATISTA, R. F. 2012. Integrative study of Brazilian anurans: geographic distribution, size, environment, taxonomy, and conservation. Biotropica 44(6):785-792.

Tubelis, D. P. 2009. Veredas and their use by birds in the Cerrado, South America: a review. Biota Neotropica 9(3):363-374.

Uetanabaro, M.; Prado, C. P. A.; Rodrigues, D. J.; Gordo, M. \& Campos, Z. 2008. Guia de Campo dos Anuros do Pantanal e Planaltos de Entorno. Cuiabá, Ed. UFMS, 192p.

Uetanabaro, M.; Souza, F. L.; Landgref-Filho, P.; Beda, A. F. \& Brandẽo, R. A. 2007. Anfíbios e répteis do Parque Nacional da Serra da Bodoquena, Mato Grosso do Sul, Brasil. Biota Neotropica 7(3):279-289.

Valdujo, P. H.; Carnaval, A. C. O. Q. \& Graham, C. H. 2012a. Environmental correlates of anuran beta diversity in the Brazilian Cerrado. Ecography 35:1-10.

Valdujo, P. H.; Silvano, D. L.; Colli, G. \& Martins, M. 2012b. Anuran species composition and distribution patterns in Brazilian Cerrado, a neotropical hotspot. South American Journal of Herpetology 7(2):63-78

Vasconcelos, T. S.; Santos, T. G.; Rossa-Feres, D. C. \& Haddad, C. F. B. 2010. Similarity of ground-dwelling anuran (Amphibia) composition among different vegetation physiognomies in a mesophytic semideciduous forest from southeastern Brazil. North-Western Journal of Zoology 6(2):275-285.

Verdade, V. K.; Valdujo, P. H.; Carnaval, A. C.; Schiesari, L.; Toledo, L. F.; Mott, T.; Andrade, G. V.; Eterovick, P. C.; Menin, M.; Pimenta, B. V. S.; Nogueira, C.; Lisboa, C. S.; Paula, C. D. \& Silvano, D. L. 2012. A leap further: the Brazilian Amphibian Conservation Action Plan. Alytes 29(1-4):27-42.

Wang, E.; Ferreira, V. L.; Himmelstein, J. \& Strüssmann, C. 2005. Amphibians and reptiles of the Southern Pantanal. In: CHANDLER, M.; Wang, E. \& Johansson, P. eds. Pantanal Conservation Research Initiative. Maynard, Earthwatch, p.30-38.

Weber, E.; Hasenack, H. \& Ferreira, C. J. S. 2004. Adaptação do modelo digital de elevação do SRTM para o sistema de referência oficial brasileiro e recorte por unidade da federação. Porto Alegre, UFRGS Centro de Ecologia. Disponível em <http://www.ecologia. ufrgs.br/labgeo>. Acessado em 29.11.2012.

Wilkison, M.; San Mauro, D.; Sherratt, E.; \& Gower, D. J. 2011. A nine-family classification of caecilians (Amphibia: Gymnophiona). Zootaxa 2874:41-64. 